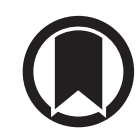

CrossMark

\title{
Clarifying the diagnosis of post- inflammatory pulmonary fibrosis: a population-based study
}

\begin{abstract}
To the Editor:
Epidemiological studies are important in defining the distribution and burden of diseases in a population. A common method of studying interstitial lung disease (ILD) epidemiology has been the analysis of insurance and billing claims databases, such as the Commercial Claims and Encounters Database and the Medicare Supplemental and Coordination of Benefits Database. These studies rely on the accuracy of International Statistical Classification of Diseases (ICD) codes to identify a patient population of interest. Several studies have described the incidence and prevalence of ILD by methodically searching ICD codes related to ILD or by using code-based algorithms [1-6].
\end{abstract}

Post-inflammatory pulmonary fibrosis (PPF) (ICD-9-CM 515) has been categorised as a general diagnostic code used by providers for IPF, an ILD characterised by progressive parenchymal fibrosis [1,7]. Cases of PPF have been variably included in studies of IPF epidemiology. The prevalence of PPF may be comparable or higher to that of IPF. For example, Coultas et al. [2] reported PPF to represent $16.7 \%$ of prevalent cases of ILDs while IPF comprised $22.5 \%$ in a population-based registry. RAGHU et al. [8] analysed a large healthcare claims database spanning the period 1996-2000 and found the prevalence of PFF to be nearly 11-fold higher than that of IPF identified by "broad case definition". To our knowledge, however, cases designated as PPF have never been fully characterised. In particular, it is unknown to what extent PPF (ICD-9-CM 515) overlaps with the diagnosis of IPF.

With approval from the Mayo Foundation Institutional Review Board (Mayo Clinic, Rochester, MN, USA), we conducted a population-based, retrospective cohort study of residents of Olmsted County, Minnesota, USA. The objectives of this study were to diagnostically characterise patients who have received the diagnosis code ICD-9 515 (PPF) and determine what portion were IPF. This study utilised the Rochester Epidemiology Project (REP), which is a medical records linkage system established in 1966 to capture healthcare data for the population of Olmsted County [9]. Persons were classified as Olmsted County residents if they had had a residential or nursing home address for $>1$ year [9].

All patients $\geqslant 18$ years of age who received the diagnosis code of PPF (ICD-9 515) and received care in Olmsted County from 1997 to 2017 were identified. A total of 4813 unique patients were retrieved. From this cohort, we analysed those who had undergone chest computed tomography (CT) imaging (1789 patients) to enable characterisation of their lung disease.

Medical records and associated chest CT studies were reviewed and the pulmonary disease was categorised based on clinical context and predominant CT chest abnormality. These categories included airway abnormalities, atelectasis, bilateral interstitial infiltrates, cysts or cavities, oedema, emphysema, focal fibrotic abnormalities, focal or multifocal infiltrates, lymphadenopathy, nodules or masses, and pleural abnormalities (table 1). We assigned a specific diagnosis whenever possible if appropriate diagnostic evaluation was performed; in other cases, only a descriptive radiological finding was assigned. IPF was diagnosed based on 2018 Fleischner criteria [10]. A diagnosis of definite or probable IPF was assigned to patients with the appropriate clinical context and CT findings (based on review of both radiology reports and actual images) suggestive of a typical or probable usual interstitial pneumonia pattern. Clinical context criteria for IPF included age $\geqslant 60$ years, absence of potentially relevant environmental or medication

@ERSpublications

Patients with post-inflammatory pulmonary fibrosis were found to be a heterogeneous population, and the vast majority did not have IPF or any form of interstitial lung disease http://bit.ly/2wcqepC

Cite this article as: $\mathrm{Vu} \mathrm{A}$, Vasireddy A, Moua T, et al. Clarifying the diagnosis of post-inflammatory pulmonary fibrosis: a population-based study. Eur Respir J 2019; 54: 1900103 [https://doi.org/10.1183/ 13993003.00103-2019]. 
TABLE 1 Diagnostic categories for patients assigned International Classification of Diseases-9 code 515 (post-inflammatory pulmonary fibrosis) ( $n=1789$ )

\begin{tabular}{lc} 
Diagnostic category & Patients \\
\hline Airway abnormalities & $83(4.6 \%)$ \\
Atelectasis & $214(12.0 \%)$ \\
Bilateral interstitial infiltrates & $177(9.9 \%)$ \\
$\quad$ Asbestosis & $2(0.1 \%)$ \\
Combined pulmonary fibrosis and emphysema & $3(0.2 \%)$ \\
Connective tissue disease-related ILD & $33(1.8 \%)$ \\
Respiratory bronchiolitis-associated ILD/DIP & $5(0.3 \%)$ \\
Hypersensitivity pneumonitis & $6(0.3 \%)$ \\
Idiopathic pulmonary fibrosis & $71(4.0 \%)$ \\
Idiopathic nonspecific interstitial pneumonia & $29(1.6 \%)$ \\
Sarcoidosis & $3(0.2 \%)$ \\
Cysts or cavities & $6(0.3 \%)$ \\
Oedema & $22(1.2 \%)$ \\
Emphysema & $49(2.7 \%)$ \\
Focal fibrotic abnormalities & $362(20.2 \%)$ \\
Focal or multifocal (nonfibrotic) infiltrates & $191(10.7 \%)$ \\
Lymphadenopathy & $23(1.1 \%)$ \\
Nodules or masses & $552(30.9 \%)$ \\
Normal parenchyma & $44(2.5 \%)$ \\
Pleural abnormalities & $66(3.7 \%)$ \\
\end{tabular}

ILD: interstitial lung disease; DIP: desquamative interstitial pneumonia.

exposure and no evidence of connective tissue disease [10]. Surgical biopsy was not required for IPF diagnosis. A second study panel member also reviewed any cases of IPF or ILD, and the final diagnosis was given based on consensus.

Among the 1789 patients assigned the ICD-9 code 515 (PPF) with CT chest imaging, 177 (9.9\%) had bilateral interstitial infiltrates consistent with ILD; only 71 (4.0\%) patients had definite or probable IPF by the 2018 Fleischner criteria. The remaining 1612 (90.1\%) patients were non-ILD cases. The largest proportion of non-ILD cases $(n=552,30.9 \%)$ manifested nodules or masses followed by focal fibrotic abnormalities $(n=362,20.2 \%)$ and atelectasis $(n=214,12.0 \%)$ (table 1). Over the study interval, there was a temporal trend to increased use of the ICD-9 code 515. For example, the number of cases designated with this code ranged 58-75 per year during the first 3 years compared to $99-178$ per year during the last 3 years. The Olmsted County population increased $\sim 1.3$-fold during this interval.

Of those with ILD other than IPF, the most common findings were connective tissue disease-related ILD $(\mathrm{n}=33,1.8 \%)$ followed by idiopathic nonspecific interstitial pneumonia $(\mathrm{n}=29,1.6 \%)$. Other diagnoses included combined pulmonary fibrosis and emphysema, hypersensitivity pneumonitis, sarcoidosis and respiratory bronchiolitis-associated ILD/desquamative interstitial pneumonia.

Our study shows that the PPF diagnosis represents a heterogeneous population, only a small portion of which had IPF or any ILD. To our knowledge, this is the first study to characterise patients assigned the diagnostic code ICD-9 code 515 (PPF) in clinical practice. The majority of our PPF patients manifested focal parenchymal abnormalities such as nodules/masses or focal fibrotic change on chest CT study. Of the 552 patients with nodules or masses, most patients $(75 \%)$ did not have a specific pulmonary diagnosis. Similarly, the vast majority $(90 \%)$ of those with focal fibrotic abnormalities did not have a specific pulmonary diagnosis other than descriptive CT findings. The diagnostic code of PPF appears to have been assigned by the clinicians or billing coders based on interpretation of radiological findings.

Our findings have implications for the use of ICD-9 code 515 (PPF) in epidemiological studies of ILD and specifically, IPF. The vast majority of patients designated as PPF did not have IPF or any form of ILD as assessed in this population-based study with 20 years of data. Olmsted County represents a unique region where medical care is provided by two health systems and longitudinal, comprehensive data can be captured. The use of the REP ensured that diagnoses were not routinely assigned from outside sources. Strict case criteria for IPF were used per Fleischner guidelines and real-world data were validated by manual chart review. 
There are limitations of this study. Our data are based on a patient population residing in the southeastern portion of Minnesota. Though the demographics of IPF patients in Olmsted County are similar to those diagnosed internationally, study findings may not be generalisable to all populations across the USA or the world [11-13]. In addition, most IPF patients did not undergo surgical biopsy, with the diagnosis assigned based on review of the clinical context and chest CT findings.

Based on our results, we conclude that only a small minority of patients assigned the ICD-9 code 515 (PPF) have IPF. In clinical practice, PPF diagnosis comprises a heterogeneous population manifesting a wide array of chest CT findings, mostly nondiffuse. Future studies assessing the features of patients designated as PPF may provide additional insights to better defining the epidemiology of IPF.

Ann Vu, Amit Vasireddy, Teng Moua, Misbah Baqir and Jay H. Ryu

Division of Pulmonary and Critical Care Medicine, Mayo Clinic, Rochester, MN, USA.

Correspondence: Ann Vu, Division of Pulmonary and Critical Care Medicine, 200 First Street SW, Rochester, MN 55905-0002, USA. E-mail: vu.ann@mayo.edu

Received: Jan 142019 | Accepted after revision: March 272019

Conflict of interest: None declared.

Support statement: This study was made possible using the resources of the REP, which is supported by the National Institute on Aging of the National Institutes of Health under Award Number R01AG034676. The content is solely the responsibility of the authors and does not necessarily represent the official views of the National Institutes of Health. Funding information for this article has been deposited with the Crossref Funder Registry.

\section{References}

1 Raghu G, Chen SY, Yeh WS, et al. Idiopathic pulmonary fibrosis in US Medicare beneficiaries aged 65 years and older: incidence, prevalence, and survival, 2001-11. Lancet Respir Med 2014; 2: 566-572.

2 Coultas DB, Zumwalt RE, Black WC, et al. The epidemiology of interstitial lung diseases. Am J Respir Crit Care Med 1994; 150: 967-972.

3 Esposito DB, Lanes S, Donneyong M, et al. Idiopathic pulmonary fibrosis in United States automated claims. incidence, prevalence, and algorithm validation. Am J Respir Crit Care Med 2015; 192: 1200-1207.

4 Farrand E, Anstrom KJ, Bernard G, et al. Closing the evidence gap in interstitial lung disease: the promise of real world data. Am J Respir Crit Care Med 2019; 199: 1061-1065.

5 Collard HR, Chen SY, Yeh WS, et al. Health care utilization and costs of idiopathic pulmonary fibrosis in U.S. Medicare beneficiaries aged 65 years and older. Ann Am Thorac Soc 2015; 12: 981-987.

6 Olson AL, Gifford AH, Inase N, et al. The epidemiology of idiopathic pulmonary fibrosis and interstitial lung diseases at risk of a progressive-fibrosing phenotype. Eur Respir Rev 2018; 27: 180077.

7 Collard HR, Ward AJ, Lanes S, et al. Burden of illness in idiopathic pulmonary fibrosis. J Med Econ 2012; 15: 829-835.

8 Raghu G, Weycker D, Edelsberg J, et al. Incidence and prevalence of idiopathic pulmonary fibrosis. Am J Respir Crit Care Med 2006; 174: 810-816.

9 St Sauver JL, Grossardt BR, Yawn BP, et al. Data resource profile: the Rochester Epidemiology Project (REP) medical records-linkage system. Int J Epidemiol 2012; 41: 1614-1624.

10 Lynch DA, Sverzellati N, Travis WD, et al. Diagnostic criteria for idiopathic pulmonary fibrosis: a Fleischner Society White Paper. Lancet Respir Med 2018; 6: 138-153.

11 Wuyts WA, Dahlqvist C, Slabbynck $\mathrm{H}$, et al. Baseline clinical characteristics, comorbidities and prescribed medication in a real-world population of patients with idiopathic pulmonary fibrosis: the PROOF registry. BMJ Open Respir Res 2018; 5: e000331.

12 Guenther A, Krauss E, Tello S, et al. The European IPF registry (eurIPFreg): baseline characteristics and survival of patients with idiopathic pulmonary fibrosis. Respir Res 2018; 19: 141.

13 Fernandez Perez ER, Daniels CE, Schroeder DR, et al. Incidence, prevalence, and clinical course of idiopathic pulmonary fibrosis: a population-based study. Chest 2010; 137: 129-137. 